\title{
Vehicle Detection Based on Laser Radar
}

\author{
Fernando Garcia ${ }^{1}$, Pietro Cerri ${ }^{2}$, Alberto Broggi ${ }^{2}$, \\ Jose Maria Armingol ${ }^{1}$, and Arturo de la Escalera ${ }^{1}$ \\ 1 Intelligent Systems Lab. Universidad Carlos III de Madrid, Spain \\ \{fegarcia, escalera, armingol\}@ing.uc3m.es \\ www.uc3m.es/islab \\ 2 VisLab.Universitá degli Studi di Parma, Italy \\ \{cerri, broggi\}@vislab.it \\ www.vislab.it
}

\begin{abstract}
This paper describes the detection of moving obstacles using laser radar in road environments. This application is designed to be implemented in further research on data fusion technologies. The developed application uses only a laser radar which provides information to sort objects according to their shape and movement. The subsequent detection and classification provide higher level tracking.
\end{abstract}

Keywords: ADAS, Intelligent Vehicles, Data Fusion, Laser Radar.

\section{Introduction}

Over the years, countless efforts have been made to decrease the number of casualties on roads. In the recent years most of these efforts have focused on developing technologies that both help and warn drivers in the event of hazardous situations. Due to recent advances in information technologies, new applications can be developed to prevent these situations. In this context, the lack of cheap and reliable sensors underlines the need to use different sensors at the same time in order to provide a reliable and accurate application.

A possible set of sensors used in data fusion applications are computer vision and radar. The reason for using these sensors is that radar provides a reliable source of possible detections in the surroundings; on the other hand, data provided by vision sensors allow the different objects detected by the laser to be classified. The application presented in this paper focuses on the detection of moving obstacles, mainly vehicles, using a laser radar integrated in the bumper of a test vehicle. Detection based on laser radar is also very useful in regions where no visual information is available and the estimations have to be done with only the laser data.

\subsection{State of the Art}

While most of the applications developed fuses frequency radar information and visual information [1, 2] and [3, in recent years laser radar are becoming more 


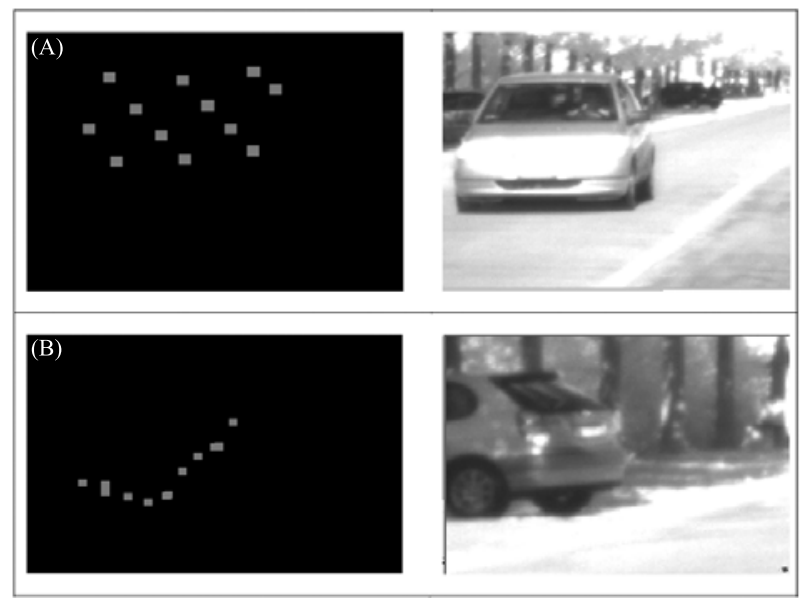

Fig. 1. Different patterns given by the laser. (A) Moving Car.(B) Parked Car.

popular 4] due to their lower cost. Laser radar also give more complete and reliable information.

The methods differ mainly in the level in which fusion is done. Low level classification converts unprocessed data from several sensors into a new set of raw data to be processed [2. Medium level fusion methods get different patterns given by the sensors (corners, shapes, colors, movements ...) and performs the classification according to them. High level fusion methods performs different classification for each sensor, and at the final stage, all classifications are checked to provide a more reliable detection [5].

The application presented focuses on the detection of moving obstacles, mainly cars, using a laser radar mounted in a vehicle bumper. Once the detection is performed it creates a log of the movement of the object. So after some time, the application is able to give an estimation about which kind of obstacle is more likely to be. This research is a first stage of a further fusion method, based on laser radar and visual information. Typically these fusion methods has some parts of the images where data is not provided by both sensors, thus the detection has to be done using only one them. Thanks to this research, an estimation of the obstacles in the surroundings can be perform, with only the information provided by the radar.

In section 2 laser radar behavior is explained. Section 3 focuses on the algorithm developed for this application. Finally, section 4 presents some results, conclusions and gives future steps .

\section{Laser Radar Behavior}

The laser radar used is the SICK LMS 211. It gives a $2 \mathrm{D}$ reading of $100^{\circ}$ around the vehicle with a $0.25^{\circ}$ resolution. To achieve this it performs 4 scans 


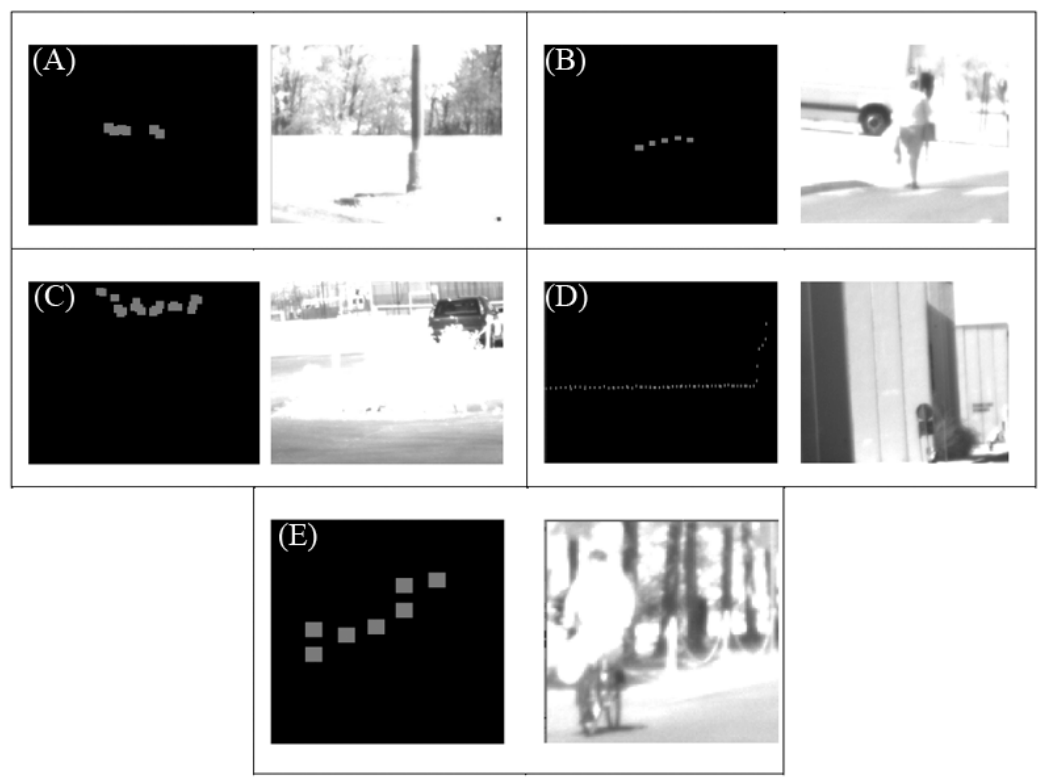

Fig. 2. Different patterns given by the laser. (A) Lamppost. (B) Pedestrian. (C) Bushes. (D) Building. (E) Bicycle.

independently which give 4 sets of spots with $1^{\circ}$ of resolution on each spot. When a moving obstacle is found, the four scans performed by the laser for a single detection appear with a variation which is proportional to the speed and direction of the detected object and the test vehicle.Hence, performing egomotion correction moving obstacles show a special pattern proportional to their movement.

A typical problem when dealing with this applications is that moving obstacles pattern given by the laser radar is very similar to other patterns that can lead to mistakes, so false positives are common. Typically false positives appears dealing with pitch movements and bushes as it is shown in Fig. 2. To avoid those false positives, filtering the detected moving obstacles is mandatory. Finally a tracking system is very useful in order to record the movement of the object along the environment. This tracking systems, also allows to detect moving obstacles even when they are not detected ( false negatives ), also a record of the movement is useful to predict their future position.

\section{Algorithm Explanation}

\subsection{Data Acquisition and Egomotion Correction}

The application receives the information from the laser and corrects it according to egomotion information. The egomotion is provided by the test vehicle via 
CAN-BUS. This information allows to correct the difference between the four consecutive scans due to test vehicle movement. After the egomotion correction, moving obstacles still appear with a variation which is proportional to the speed and direction of the detected object only (see fig. 1.A); thus by taking advantage of this behavior, it is possible to know whether an obstacle is moving or not.

\subsection{Low Level Classification}

The sets of points are separated, according to the distance between them, into different obstacles and their shapes are estimated. The shape is estimated by merging the points for each obstacle into a polyline that defines its shape. A low level classification is made taking into account the shape of the obstacles, moving obstacles may be differentiated thanks to their serrated shape. The sets of obstacles that are differentiated are:

- L Shape Obstacle. Obstacle with the specific L shape that typically is given by parked car.

- Possible Pedestrian. For obstacles with a small size that could represent wether pedestrian or typical road object that could be detected, like lamppost or signs.

- Fixed Obstacle. Obstacles that does not fit in all the rest possible obstacles are labeled as fixed obstacles.

- Road Border. When the size and position of the obstacle is suitable to fix in this kind of obstacle it is labeled as road border. Also a high number of possible pedestrian obstacles parallel to the movement of the car which could mean that there is railguards or milestones in the road are labeled as road borders.

- Moving Obstacle. This is the main part of the algorithm due to their special and interesting shape.

\subsection{Moving Obstacle Detection}

The serrated shape that presents moving obstacles and the divergence between the points, make possible to detected them and their trajectory and speed estimated. Some constraints in the speeds or movements (impossible speeds, accelerations and shapes that does not match with cars shape ) are mandatory to avoid false positives.

Where $\mathrm{T}=13 \mathrm{msecs}$, which is the period of a single scanner rotation.

The speed can be computed three times each complete scan, so it is also possible to calculate the acceleration of the moving obstacle by calculating the difference in the calculated speeds. These information makes possible differentiate between real moving cars and false positives. The acceleration and speed calculated are not accurate due to the $0,25^{\circ}$ divergence between two rotations, but precise enough to avoid those false positives. 


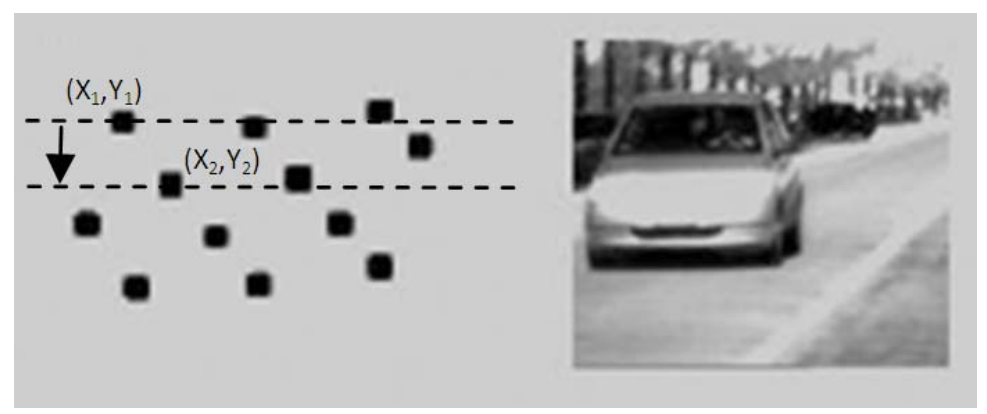

Fig. 3. Divergence between points can be used to detect false positives

$$
v=\frac{\sqrt{\left(x_{1}-x_{2}\right)^{2}+\left(y_{1}-y_{2}\right)^{2}}}{T}
$$

\subsection{Tracking Stage}

After the low level classification, a tracking stage is performed in order to detect similar objects in the following laser scan. This tracking is done with both the information provided by the low level detection in the current scan and the previous tracking.

The tracking stage computes the speed of the car and calculates the position for the next scan. The moving obstacle is searched within a given window which is proportional to the size and shape of the moving obstacle. If a moving obstacle is detected, the speed is actualized according to the difference in the position of the last two frames and the low level classification is stored. This way the estimated speed in this stage is more accurate than lower level speed estimation which has the problem of the $0.25^{\circ}$ divergence for each rotation. After some consecutive moving obstacle detections a higher level estimation is done using a voting scheme based in the latest low level estimations. The longer the obstacle is detected, the more reliable the classification.

Low level false positives are one of the most challenging situations in this stage. Tracking algorithm has to deal with these detections and avoid tracking them. Impossible movements and big size changes are detected and labeled as false positives,thus tracking is not performed for these obstacles.

\section{Results, Conclusions and Future Steps}

\section{$4.1 \quad$ Results}

Test has been performed under real conditions to check the reliability of the system. 


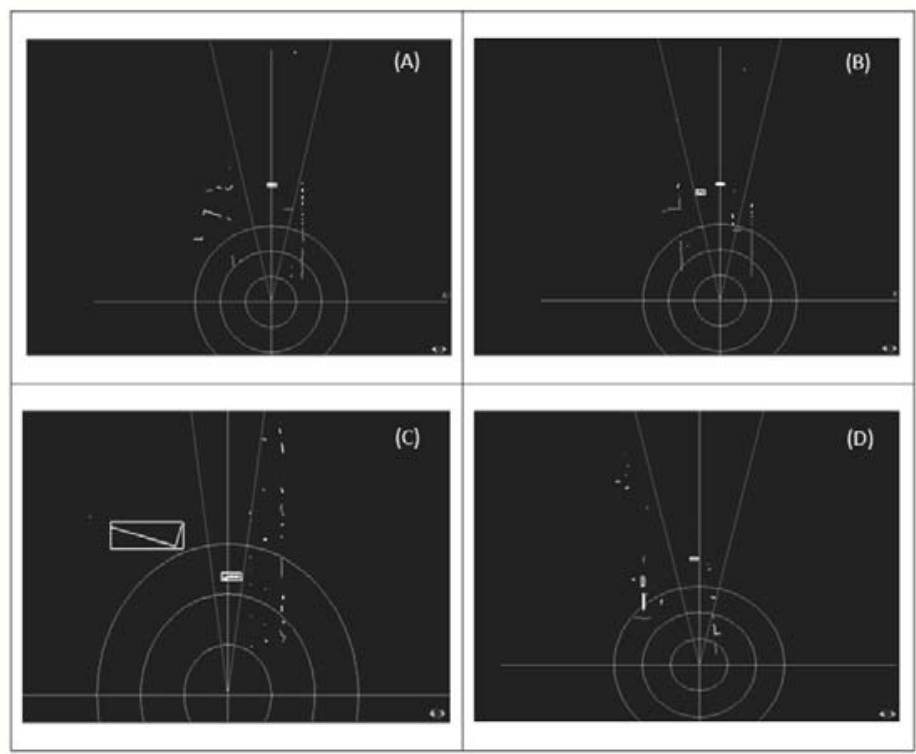

Fig. 4. Results. (A) Real traffic with a car ahead. (B) Two cars in opposite directions. (C) A truck and a bus in a crossroad. (D) A car ahead and two false positives.

Low Level Detection Test. two different Test were performed, the first where perform with the test vehicle stopped. the main purpose of the test was to check the viability of the algorithm avoiding possible problems due to egomotion correction. The results obtained were $80 \%$ positive detections for a single car in an approach movement for a distance closer to 48 meters, and 36 meters if the car is separating from the laser. In closer distances the detection percentage is higher. The results were very positive since this percentage could be increased by adding the tracking stage which, once the first detection is performed, can track the vehicle even when it is not detected.

Moving test were performed and egomotion correction included in the application. The results obtained were very similar to previous results. The test showed that egomotion corrections plays an important role in the detections of the surroundings. While the data provided by the test vehicle related to the velocity resulted reliable, the yaw angle were not so accurate. This problem lead to some false positives when lateral movement were involved, mainly curves. Also pitch movements when breaking or accelerating caused some false positives.

Complete Algorithm Test. Tracking resulted to be very useful since once the vehicle were detected in could be tracked until it disappears. The main problems in this stage were caused by low level false positives which should be avoided. 


\subsection{Conclusions}

It has been proved that vehicle detection can be done using only laser radar information. Information provided by this applications are not limited to shapes, it also can be detected speeds, accelerations and movement. A tracking stage can help to avoid missdetections. Finally, it also has been proved that, when dealing with real situations, egomotion correction plays an important role and need to very accurate to avoid false positives.

\subsection{Future Steps}

The applications represents the first step of a complete fusion algorithm which includes visual and laser radar information. Future step fuses this application with visual information algorithms. Visual information can avoid some false positive due egomotion correction errors, by checking the detections using visual information. Egomotion correction has to be improved.

\section{Acknowledgements}

This application has been developed by members of VisLab [6] and Intelligent System Lab 7] as an Integrated Action between the two groups. The system has been tested on recorded sequences provided by the test platform "Grandeur" 4. This research has been developed in the scope of scanning laser radar-based pedestrian detection project funded by Mando (South Korea).

This work also was supported in part by the Spanish Government through the CICYT projects VISVIA (Grant TRA2007-67786-C02-02) and POCIMA (Grant TRA2007-67374-C02-01).

\section{References}

1. Ofer, A.S., Mano, O., Stein, G.P., Kumon, H., Tamatsu, Y., Shashua, A.: Solid or not solid: Vision for radar target validation. In: IEEE Intelligent Vehicles Symposium Proceedings, pp. 819-824 (2004)

2. Steux, B., Laurgeau, C., Salesse, L., Wautier, D.: Fade: a vehicle detection and tracking system featuring monocular color vision and radar data fusion, June 2002, vol. 2, pp. 632-639 (2002)

3. Alessandretti, G., Broggi, A., Cerri, P.: Vehicle and guard rail detection using radar and vision data fusion. IEEE Transactions on Intelligent Transportation Systems 8(1), 95-105 (2007)

4. Broggi, A., Cerri, P., Ghidoni, S., Grisleri, P., Jung, H.G.: Localization and Analysis of Critical Areas in Urban Scenarios. In: Procs. IEEE Intelligent Vehicles Symposium 2008, Eindhoven, Netherlands, June 2008, pp. 1074-1079 (2008)

5. Hofmann, U., Rieder, A., Dickmanns, E.D.: Radar and vision data fusion for hybrid adaptive cruise control on highways. In: Schiele, B., Sagerer, G. (eds.) ICVS 2001. LNCS, vol. 2095, pp. 125-138. Springer, Heidelberg (2001)

6. http://www.vislab.it

7. http://www.uc3m.es/islab 